\title{
L'ethos collectif des guerrilla gardeners à Montréal : entre conflictualité et inclusion
}

The Collective Ethos of Montreal Guerrilla Gardeners: Between Conflict and Inclusion

\section{Anna Giaufret}

\section{(2) OpenEdition}

\section{Journals}

Electronic version

URL: http://journals.openedition.org/aad/1978

DOI: $10.4000 /$ aad. 1978

ISSN: 1565-8961

\section{Publisher}

Université de Tel-Aviv

\section{Electronic reference}

Anna Giaufret, "L'ethos collectif des guerrilla gardeners à Montréal : entre conflictualité et inclusion », Argumentation et Analyse du Discours [Online], 14 | 2015, Online since 09 April 2015, connection on 23 September 2019. URL : http://journals.openedition.org/aad/1978 ; DOI : 10.4000/aad.1978

This text was automatically generated on 23 September 2019.

\section{(i) ()}

Argumentation \& analyse du discours est mis à disposition selon les termes de la licence Creative Commons Attribution - Pas d'Utilisation Commerciale - Pas de Modification 4.0 International. 


\title{
L'ethos collectif des guerrilla gardeners à Montréal : entre conflictualité et inclusion
}

\author{
The Collective Ethos of Montreal Guerrilla Gardeners: Between Conflict and \\ Inclusion
}

Anna Giaufret

\section{Introduction : la Guerrilla Gardening}

1 La Guerilla Gardening (ou guérilla jardinière, guérilla potagère, guérilla du jardinage) - que nous désignerons dorénavant par le sigle GG - est un phénomène peu étudié. Le mouvement fait partie de la constellation de formes de mobilisation citoyenne que semblent aujourd'hui préférer les activistes, qui délaissent de plus en plus les formes de lutte traditionnelles :

La désaffection partisane et associative a en ce sens parmi ses corollaires la radicalisation vers un type d'investissement politique qui n'a pas pour finalité la quête du pouvoir au sein des institutions existantes, mais vise plutôt à préfigurer dans des expériences concrètes la nouvelle société à construire (Sarrasin, Kruzynski, Jeppesen \& Breton $2012: 142$ ).

2 Née, sous sa forme actuelle, à New York dans les années soixante-dix (elle s'appelait alors green guerrilla), la GG s'est aujourd'hui répandue dans le monde, notamment en Grande Bretagne, dans les pays d'Amérique du Nord, y compris le Québec.

Les rares publications sur le sujet s'inspirent, pour la présentation et l'analyse du mouvement, des deux grands textes fondateurs qui ont contribué à sa construction discursive, à savoir David Tracey $2007^{1}$ et Richard Reynolds $2008^{2}$ : c'est précisément par la narration de l'histoire du mouvement, par son positionnement dans les courants écologistes et altermondialistes, par la description de ses liens de filiation que l'identité de la GG se dessine. Il ne semblerait donc pas qu'il existe, à l'heure actuelle, un discours véritablement extérieur à la GG, même dans les publications à caractère scientifique, 
dont les seules sont la thèse de doctorat (canadienne) en géographie de Annie Crane $(2011)^{3}$ et l'article qui en est issu (Crane, Viswanathan, Withelaw 2013). Les auteurs de ce dernier citent seulement six travaux portant sur la GG (Pinder 2005, Tracey 2007, Hou 2010, McClintock 2010, Ryan 2010, Zanetti 2010) parmi lesquels les seuls ouvrages entièrement consacrés à la GG sont l'un des manifestes du mouvement (Tracey 2007) et le mémoire de Master de Olly Zanetti (2010), un activiste anglais qui collabore avec Richard Reynolds.

La définition du mouvement reste complexe. Annie Crane (2011), dans la seule contribution scientifique d'une certaine ampleur entièrement consacrée à la GG, écrit :

For the purposes of this study, guerrilla gardening will refer to gardening or planting in an unadministered way in an urban space. By unadministered I mean that guerrilla gardening is not performed by a specific state or official organization. Instead, it is an individual or a group of individuals who frequently operate spontaneously, anonymously and voluntarily. While there may or may not be unlawful activity the lack of explicit permission and unexpected nature allow for guerrilla gardening to be a form of spatial intervention (Crane $2011: 17$ ).

Encore selon Crane (2011), la GG est souvent classée parmi les mouvements d'agriculture urbaine au même titre que le jardinage communautaire, quoiqu'elle s'en démarque par son caractère revendicatif, transgressif et interventionniste. Elle s'approche aussi du street art par ses aspects esthétiques (à Montréal notamment certains artistes pratiquent parallèlement et conjointement les deux $)^{4}$. Crane souligne aussi le caractère très géographiquement situé de la GG : non seulement les activistes sont liés à une ville et à une zone urbaine, mais la définition même de la GG change selon sa localisation : un groupe ou un projet de GG sont « highly contextual with some overriding themes based on the goals and emerging conceptual understanding of guerrilla gardening » (Crane $2011: 18)$.

\section{La Guerrilla Gardening à Montréal}

Quoiqu'il soit difficile de tracer un panorama des activités relevant de la GG dans la ville de Montréal, à cause de la nature foncièrement "secrète " du mouvement, il est toutefois possible d'en identifier certaines grandes tendances. Il semble que la GG s'implante dans la ville depuis 2008 (les premiers textes qui la mentionnent remontent à cette date) grâce notamment à l'activité d'individus qui mettent en place des projets de quartier, susceptibles ensuite de rassembler autour d'eux d'autres personnes et parfois de créer des groupes.

7 Il est possible d'identifier, parmi les acteurs sociaux, plusieurs profils :

- Les activistes. Certains sont des fréquentateurs habituels du forum guerrillagardening.org ${ }^{5}$ et des activistes chevronnés. On retrouve parfois leurs noms (ou plus souvent leurs pseudonymes) dans la presse. Les plus assidus (et connus) sont Luc 158 et Univert (du groupe "Criminels verts »). Ils préfèrent garder l'anonymat et, s'ils appartiennent à des groupes, ceux-ci ne possèdent pas de pages web. Il s'agit des guérilleros du jardinage purs et durs, qui organisent une attaque puis disparaissent ;

- Les groupes: Occupy gardens Montréal ${ }^{6}$; La fosse aux lionnes, Criminels verts, Guérilla Gardening dans le Mile End; Guerrilla Gardening Montréal. La plupart sont mentionnés dans les forums ou les médias mais ne laissent souvent aucune trace de leurs actions sur le web ; 
- Les catalyseurs : des individus qui lancent un projet tous seuls puis sont rejoints par d'autres habitants du quartier : Laurent Richer (rue des Ecores) ; Trixi Rittenhouse (rue Labadie, puis Plateau) ; Emily Wilkinson et Torsten Hermann (St-Henri), etc. ;

- Les artistes: Emily Rose Michaud ${ }^{7}$, Glen Lemesurier ${ }^{8}$, Donovan King ${ }^{9}$, Peter Gibson ${ }^{10}$, Gina Badger ${ }^{11}$.

Le caractère flou et changeant des contours du mouvement et sa proximité avec le monde de l'agriculture urbaine et avec celui de l'art urbain font en sorte qu'il existe également des entités qui ne se concentrent pas uniquement ou ouvertement sur la GG, mais la côtoient. Il s'agit de centres institutionnels (le Centre d'écologie urbaine de Montréal qui propose des promenades urbaines pour voir les résultats d'actions de GG) ${ }^{12}$, des collectifs de recherche (le CRAPAUD - le Collectif de recherche sur l'aménagement paysager et l'agriculture urbaine durable - de l'Université du Québec à Montréal ; le CRAC, Collectif de recherche sur l'autonomie Collective) ${ }^{13}$ ou encore des expositions et initiatives muséales (exposition Actions: comment s'approprier la ville au Centre Canadien d'Architecture de Montréal, 2008-2009). ${ }^{14}$

Or, la GG est un terrain d'enquête intéressant car peu d'études lui ont été consacrées, notamment dans une perspective d'analyse du discours, et c'est notamment cette dernière qui peut contribuer à mieux le définir. Le mouvement de la GG - avec son caractère à la fois décentralisé et ancré à chaque fois dans un territoire précis et restreint (la ville, mais plus souvent le quartier), son caractère souterrain, secret, voire clandestin, ainsi que ses activités fondées sur l'anonymat, l'effet de surprise) pose plusieurs problématiques, notamment dans le contexte montréalais dans lequel ses activistes doivent se positionner par rapport au clivage francophone/anglophone. En effet, ce mouvement, centré sur une proximité géographique, rassemble à Montréal des activistes francophones et anglophones, et donne lieu à des relations et à une communication entre les deux communautés linguistiques. Ainsi, je souhaite vérifier l'hypothèse que l'origine très nettement anglaise ou canadienne-anglaise du mouvement, puisse exercer une pression sur les énonciateurs et les pousser à se positionner discursivement afin de construire (ou non) un lien entre leur identité collective langagière et celle du mouvement.

Ma contribution va donc analyser la construction discursive de l'identité du mouvement de la GG à Montréal, telle qu'elle se réalise à la fois dans les discours produits de l'intérieur du mouvement, mais aussi dans ceux qui sont produits de l'extérieur sur le mouvement.

\section{Cadrage théorique et outils d'analyse : ethos et locuteur collectif}

11 Se focalisant sur l'identité discursive du mouvement, mon analyse va utiliser deux notions fondamentales: celle d'ethos - ethos collectif en l'occurence - et celle de locuteur collectif. La notion d'ethos a été abordée plusieurs fois par l'analyse du discours et la théorie de l'argumentation. Je retiendrai ici la définition qu'en donne Ruth Amossy : «l'image [de l'orateur] construite dans le discours » (1999:154). Quant à la notion d'ethos collectif, je renvoie à Ruth Amossy (2010) et Eithan Orkibi (2008) pour ce qui est de l'image de soi que projette un groupe, particulièrement un mouvement social de contestation, tel que le nôtre. C'est en effet par des stratégies rhétoriques de 
construction de l'identité collective et de polarisation (stigmatisation de l'ennemi) que l'identité du groupe se construit (Orkibi 2008:3). Je retiens la définition suivante d'ethos collectif: «image de soi d'un groupe : une image mobilisée ou reflétée dans la parole de l'individu appartenant à ce groupe [...] mais aussi une image de soi collective qui est exprimée par un groupe, à savoir l'ethos du locuteur collectif» (Orkibi 2012 : 22).

Or, il semble particulièrement pertinent dans notre cas, d'intégrer aussi la notion élaborée par Patrick Charaudeau (2005:90) d'un ethos collectif hétéro-attribué, à savoir la projection sur un groupe d'une image sociale identitaire, artificiellement homogène, fondée sur des aprioris et des stéréotypes, de la part de ceux qui ne lui appartiennent pas : "L'ethos collectif correspond à une vision globale, mais à la différence de l'ethos singulier, il n'est construit que par attribution apriorique, attribution d'une identité émanant d'une opinion collective vis-à-vis d'un groupe autre» (Charaudeau 2005 : 90). Charaudeau envisage ici l'ethos comme une construction extérieure à la source énonciative qui le porte. Cette conception me semble surtout intéressante lorsqu'elle est employée à côté de celle d'ethos auto-attribué, c'est-à-dire au sens que lui donne Ruth Amossy (1999).

13 Ensuite, il est utile de rappeler ici une autre notion, celle d'ethos préalable (Amossy 1999) ou prédiscursif (Maingueneau 1999) : l'image publique d'un orateur que celui-ci apporte avec lui avant la phase discursive. Cet ethos préalable est lui aussi issu principalement de discours, en particulier celui des médias. Il correspond donc en grande partie à l'ethos hétéro-attribué. C'est précisément du décalage, de l'interaction, voire du conflit entre les deux ethè (auto- et hétéro-attribués) que se construit, au fil des discours, l'image des mouvements sociaux qui n'ont de cesse de se regarder dans le miroir que leur tendent d'autres discours, notamment de nature médiatique.

14 C'est donc à la fois sur l'ethos collectif discursif auto-attribué par les activistes de la GG (qui se doit de composer avec un ethos prédiscursif faible - à cause de la nouveauté du mouvement - mais tout de même présent, car la GG se construit comme mouvement dans sa relation à d'autres mouvements préexistants et bien connus) et sur l'ethos collectif hétéro-attribué à la GG de l'extérieur que je vais travailler.

15 L'ethos collectif me conduit à réfléchir sur sa source énonciative: les locuteurs collectifs, définis par Bernard Gardin dans le Dictionnaire d'analyse du discours (2002: 352) comme " Auteurs des discours émanant de partis, syndicats ou autres groupes organisés dont le chercheur pose, sous certaines conditions de production, qu'ils sont représentatifs du groupe ". Il semble par ailleurs utile, dans le contexte de la GG, d'intégrer les propos du sociolinguiste urbain Thierry Bulot, qui nous rappelle que : « Il est concevable de parler de locuteur collectif pour toute forme discursive porteuse de rapports sociaux" (2001: 10, note 11). Or, les rapports sociaux construits par nos locuteurs collectifs sont des relations fondées sur l'appartenance au même groupe (la GG), au même espace géographique (Montréal, souvent le quartier) et à un espace linguistique traversé par le clivage anglais/français, au point qu'on a pu parler de "ségrégation linguistique" (Elke 2002: 145) ${ }^{15}$. Il est également bien connu que l'élément langagier constitue une composante identitaire fondamentale pour la communauté francophone québécoise. Non seulement une langue porte avec elle une image de soi plus ou moins valorisante ou valorisée ${ }^{16}$, mais chaque locuteur doit, dans le contexte montréalais, se positionner par rapport à la langue : par le choix du français et de l'anglais d'abord, par la monstration d'une maîtrise langagière, par d'éventuels commentaires épilinguistiques. 
Mon travail va donc porter sur l'analyse d'un corpus multiforme, au sein duquel je vais me pencher en particulier sur la relation entre ethos collectif et ethos hétéro-attribué et leurs mises en scène discursives, en m'appuyant notamment sur la question du clivage linguistique français/anglais.

\section{Constitution du corpus}

17 Mon corpus se compose d'un ensemble de textes numériques (blogues, forums, pages web) identifiés sur le web canadien par une recherche de l'expression "guerrilla gardening " et de ses synonymes (ce qui constitue le champ discursif de la guérilla potagère au Canada et au Québec) : nous n'avons ensuite retenu que les pages qui traitent d'activité ou d'activistes liés à la GG sur l'île de Montréal.

Ces textes sont hétérogènes à plusieurs titres :

- Leur nature : textes monologaux de type manifeste ou présentation des groupes ou des activités, etc. vs textes dialogaux de type forum.

- Les énonciateurs, les destinataires, les buts : des énonciateurs et des destinataires différents (textes hétéro-adressés, auto-adressés), ainsi que des buts différents (argumenter, convaincre, séduire, inciter à l'action) donnent lieu à des stratégies de construction de l' ethos et de l'identité très différentes ${ }^{17}$.

- La langue : les textes des mouvements, surtout les messages des forums, présentent un mélange de français et d'anglais. Le corpus presse et le corpus blogues sont composés de textes écrits dans l'une ou l'autre des deux langues.

Pour schématiser, les textes du corpus peuvent être classés en cinq sous-corpus :

- Corpus forum (2007-2013) : textes provenant du forum guerrillagardening.org montréalais dans lesquels les activistes discutent et organisent leurs actions. Il s'agit de textes dialogaux, autoproduits et autoadressés à l'intérieur du groupe. Ils sont donc homogènes selon l'énonciateur, le destinataire et la nature, mais hétérogènes du point de vue linguistique : les guérilleros montréalais, en effet, s'expriment sur un forum bilingue. Cela nous parait signifier une diminution du phénomène que la sociolinguistique avait appelé « ségrégation linguistique » des communautés anglophone et francophone à Montréal ;

- Corpus presse et médias (2008-2013) : articles parus dans la presse anglophone et francophone ;

- Corpus activistes : textes présents sur les pages de groupes ou d'activistes, pas toujours datés ;

- Corpus blogues : textes disponibles sur des pages persos et des blogues qui ne relèvent ni de la presse ni d'activistes, pas toujours datés ;

- Corpus projets institutionnels : textes disponibles sur les sites d'institutions (municipalité de Montréal, etc. ) appartenant généralement au domaine de l'agriculture urbaine.

Pour l'analyse des données, je vais d'abord présenter celles qui concernent les textes sur la GG, dans lesquels j'utiliserai la notion d'ethos hétéro-attribué. Il est par ailleurs évident que ces textes sont constitués d'un feuilleté énonciatif stratifié, puisqu'ils relatent souvent les propos d'activistes. Je n'aborde pas, dans le cadre de cette contribution, les questions d'hétérogénéité énonciative. Je passerai ensuite aux textes $\mathrm{du}$ forum des activistes et des pages Facebook des groupes. 


\section{L'ethos hétéro-attribué de la Guerrilla Gardening}

21 Je commence mon analyse par prendre en compte les traces discursives de l'ethos collectif hétéro-attribué dans les textes médiatiques, car ceux-ci sont les plus immédiatement disponibles pour le citoyen lambda. Toutefois une certaine polyphonie de ces textes laisse entendre les voix des activistes, notamment sur la question du nom (qui ouvre cette section) et qui est en quelque sorte négocié entre l'intérieur et l'extérieur du mouvement. J'aborde ensuite la définition de la GG (l'aspect dénotatif), pour finir avec une analyse de ses aspects connotatifs et de la scénographie mise en place par les théoriciens du mouvement.

\subsection{La question de la dénomination}

22 La première question est celle, terminologique, de la dénomination du mouvement. L'acte de nommer, acte énonciatif par excellence, marque à la fois l'individuation de l'objet nommé (qui se distingue donc d'autres objets semblables) et l'affirmation de son existence. Par ailleurs, la dénomination guerrilla gardening est porteuse d'une mémoire discursive et, en tant que double emprunt (de l'espagnol et de l'anglais), d'une caractérisation linguistique ${ }^{18}$. Cette nébuleuse dénotative et connotative nous semble contribuer de manière importante à la composition de l'ethos collectif.

Une recherche avec Ngram Viewer montre une lexicalisation très claire du lexème guerrilla gardening, qui commence à être attesté dans le corpus Google Books autour de 1982, augmente rapidement en 1995 et explose littéralement vers l'an 2000 avant de commencer à ralentir vers 2004. Si les résultats de Ngram Viewer pour l'anglais s'arrêtent en 2008, pour le français cette recherche est malheureusement impossible à cause de la taille réduite du corpus ${ }^{19}$, mais peut-être aussi à cause de la prolifération de dénominations équivalentes qui fragmentent excessivement le champ dénominatif.

La question terminologique de la nomination est posée par certains textes du corpus, introduisant des commentaires métalinguistiques qui évoluent diachroniquement. Si en 2008 c'est la notion même de GG qui est taxée de xénisme, en 2011 le même quotidien emploie côte à côte la dénomination française et son équivalent anglais (nous soulignons) :

Guerrilla gardening, donc. Un concept typiquement anglo-saxon, avance Ismael Hautecoeur, chargé de projet des Jardins sur les toits à l'ONG Alternatives, qui ne connaît pas d'équivalent français à l'expression. Guerilla potagère, peut-être? (Le Devoir, 19 avril 2008) ${ }^{20}$

Certains décident donc de sauter la clôture: c'est la guérilla du jardinage, ou " guérilla gardening ", un mouvement qui se répand dans plusieurs grandes villes du monde. (Le Devoir, 8 août 2011) ${ }^{21}$

Le problème de l'absence d'un équivalent français, posé en 2008, est rapidement résolu par un riche paradigme dénominationnel : non seulement le mot guérilla peut être écrit à la française (avec un accent et un seul $r$ ) ou non, être féminin ou masculin, mais il peut être accompagné de différents déterminants (jardinière, $d u$ jardinage, potagère), voire remplacé par résistance (ce qui introduit une modulation intéressante du point de vue).

Cette prolifération d'appellations en français montre bien une hésitation : d'une part la résistance québécoise à l'anglais dans les textes médiatiques incite ceux-ci à ne pas 
utiliser la dénomination anglaise, alors que c'est la seule qui se soit stabilisée, d'autre part le manque d'implantation d'un terme unique semble suggérer une phase encore primordiale de l'implantation du mouvement lui-même chez les francophones.

\subsection{La spécificité de la GG à Montréal}

27 Étroitement liée à la question précédente il y a celle de la place de Montréal dans le panorama canadien de la GG : si le mouvement s'enracine en ville, cela correspondrait à une appropriation et en quelque sorte à une intégration de cette «notion typiquement anglo-saxonne». Encore faudrait-il savoir qui la pratique: les anglophones ou les francophones? Sur ce point la presse est très discrète: on peut imaginer qu'il s'agit principalement d'anglophones en regardant les noms des activistes (un critère peu fiable au Canada et surtout à Montréal).

Au-delà de la diversité des opinions fournies dans les journaux, il est possible d'identifier une tendance de la presse francophone à limiter l'importance du phénomène, alors que la presse anglophone semble pencher davantage vers la reconnaissance d'une activité plus intense, dont elle souligne parfois l'aspect illégal et dangereux, mais aussi le caractère pancanadien :

Si on fait bien sûr abstraction du cannabis planté illicitement dans les champs de maïs, le guerrilla gardening, au dire de connaisseurs, serait au Québec un mouvement très marginal. Isabelle Grégoire est d'un autre avis, affirmant que cette philosophie est à la source des jardins collectifs, aujourd'hui plébiscités par les instances gouvernementales. (Le Devoir, 19 avril 2008) 22 $^{22}$

Car l'idée de guérilla du jardinage ne semble pas prendre racine à Montréal ou Québec, même si les terrains vagues et autres occasions ne manquent pas. Quelques événements Facebook, un peu de bouche-à-oreille, mais c'est tout. Étrangement, Toronto, Victoria et Vancouver semblent beaucoup plus enclins à entrer dans le mouvement. (Le Devoir, 8 août 2011) ${ }^{23}$

In Montreal, the guerrilla gardening movement is just taking root. Last fall, on Thanksgiving weekend, a group calling itself "criminels verts" met outside a metro station. [...] Technically, their activities are illegal. [...] Maxime Chagnon, a city of Montreal spokesman, says he's heard reports of guerrilla gardeners at work but no one has ever been fined as far as he knows. (Canada.com from Montreal Gazette, 26 mai 2008) $)^{24}$

Montreal is host to a wide variety of unconventional gardening techniques that King discovered in his search for green spaces after moving to the city. [...] Covert gardening activities certainly have the appeal of doing something risky. (Forget the box, 17 août 2010) $)^{25}$

Le contraste entre les textes en anglais et les textes en français est significatif : alors que pour la presse francophone la GG reste "très marginal[e] à Montréal », les anglophones affirment quant à eux, dans un texte par ailleurs antérieur au précédent, qu'elle est " just taking root ». Il est tout particulièrement intéressant de comparer ce dernier énoncé (2011) avec «ne semble pas prendre racine à Montréal»(2008): la même métaphore végétale est utilisée, alors que les modalisations du verbe expriment deux sens opposés. Le commentaire d'Isabelle Grégoire, qui évacue entièrement le côté intervention illicite de la GG en la réduisant à une "philosophie [...] à la source des jardins collectifs », nous mène à la question de la scénographie (Maingueneau 1999). 


\subsection{Guerre et paix}

30 Montréal se double ici d'un deuxième clivage entre une scénographie guerrière et une scénographie ${ }^{26}$ pacifiste. En effet, les énonciateurs des manifestes du mouvement, David Tracey et Richard Reynolds, se présentent tantôt par une scénographie de guerrier, tantôt par une scénographie de pacifiste bucolique. Ce phénomène ne se manifeste pas seulement sur le plan du lexique mais surtout sur celui des voix de l'autorité qui sont convoquées et qui appartiennent à la fois au monde du pacifisme (le Dalaï-Lama, Gandhi) et à la tradition nord-américaine issue de Thoreau (retour à la nature et désobéissance civile), et à la stratégie militaire (Mao Tsé-Toung, Che Guevara, mais aussi Henry Kissinger).

Cette double scénographie semble se décliner en relation étroite avec l'appartenance linguistique : en effet, alors que la presse anglophone souligne davantage l'aspect illégal et dangereux des activités menées par les guérilleros du jardinage, les francophones prennent plutôt leurs distances et préfèrent une vision irénique de la GG ou se tournent vers d'autres formes d'agriculture urbaine, tout en soulignant la précocité de l'activisme des Montréalais dans ce domaine. En somme, une vision peu agressive de la GG (certains activistes agissent en plein jour selon La Presse du 30 juillet 2008) ${ }^{27}$ et une adaptation locale moins revendicative et plus domestiquée :

Avec son blogue Le pouvoir aux pousses, l'artiste montréalaise Emily Rose Michaud est bien connue dans la communauté locale disséminée des guérilleros du jardinage. Une appellation qu'elle n'affectionne pas particulièrement. "Je connais des gens qui pratiquent le guerrilla gardening à Montréal depuis longtemps, mais ce n'est que récemment que j'ai su que ce type d'actions porte ce nom un peu agressif » (Canoe, sans date) ${ }^{28}$.

Cette version peu agressive de la GG plaît même aux institutions -

À Montréal, on semble proche d'une collaboration entre la municipalité et les jardiniers activistes [...] (Micasa, sans date) ${ }^{29}$

- au point que le Centre d'écologie urbaine de Montréal organise des promenades guidées le long des parcours de la GG :

Découvrez des initiatives insoupçonnées pour verdir Montréal! Le départ se fait à la Maison de l'Amitié pour en apprendre davantage sur le jardin communautaire qui s'y trouve, ensuite le parcours visitera des ruelles vertes du secteur et des toits verts, des jardins de " guérilla jardinière ", et d'autres projets d'urbanisme écologique ${ }^{30}$.

Même l'Université du Québec à Montréal a un groupe de recherche qui milite en faveur de la $G^{31}$, le CRAPAUD (Collectif de recherche en aménagement paysager et en agriculture urbaine durable) ${ }^{32}$, et les Muséums Nature de Montréal ont organisé un lancer de «bombes vertes », qu'on dénomme significativement «balles de vie » ${ }^{33}$.

Il est aussi intéressant de souligner que la GG intéresse à Montréal même les promoteurs immobiliers, censés être les pires ennemis des espaces verts :

I know, I know! This is supposed to be a blog about real estate. Truth be told, I'm fascinated by where we live and how we live, regardless of whether we own it or not.

That's why the concept of guerrilla gardening appeals to me. I like the idea of people sowing seeds and planting seedlings in public spaces for no other reason than the joy of watching something grow amid the squalor and neglect of some god-forsaken traffic island, back alley or hyrdo pylon ${ }^{34}$ (MontReal Estate, 6 avril 2009). 
est donc possible de conclure cette première partie en affirmant que l'ethos hétéroattribué au mouvement de la GG présente des caractéristiques différentes selon que son image est construite par les anglophones ou les francophones: ces derniers ont en effet, surtout dans un premier temps, des difficultés à intégrer le mouvement qu'ils perçoivent comme un élément étranger, de par son origine anglo-américaine et de par son caractère agressif et illicite. Le flottement dénominatif et la définition même du mouvement (plus centrée sur son caractère illicite chez les anglophones, davantage axée sur le consensus chez les francophones) semblent mettre l'accent, dans les deux communautés, sur les connotations différentes, voire opposées, qui émergent des manifestes du mouvement : la guerre d'un côté, le pacifisme de l'autre.

\section{Ethos auto-attribué}

Passons maintenant à l'analyse des textes produits par les activistes, où l'on va rechercher des observables permettant de réfléchir sur l'ethos collectif du mouvement de la GG.

\subsection{Le forum des activistes : ethos linguistique, ethos dit/montré, et scénographie}

Dans le forum des activistes, sur un total de 56 messages pertinents ${ }^{35}$ postés entre 2008 et 2013, 24 sont en français, 27 en anglais et cinq bilingues. Certains intervenants, tel que le dénommé Flatapouette sont très à l'aise dans les deux langues et participent aux fils de discussion en anglais et en français, parfois même en postant deux messages de suite dans deux langues différentes en réponse à un message bilingue. Les messages bilingues peuvent contenir le même texte dans les deux langues, parfois en version résumée, ou contenir un très bref segment dans l'autre langue, tel le post de Richard _001 (Reynolds ?) qui écrit: "More info ici ». La réponse d'un activiste à un message précédent dans la langue de l'autre (accompagné parfois d'excuses pour la mauvaise qualité de la langue), déclenche un besoin de l'interlocuteur de faire la même chose : s'il estime que sa maitrise de la langue n'est pas assez bonne, il va utiliser des outils tels que Google translate (Lucien Samary) ${ }^{36}$.

Pour ce qui est des questions d'ethos linguistique, l'exemple le plus éclairant est celui d'Univert, qui intitule son message de présentation:

Un quebeco-franco " gardener » d'amerique du nord, francais! (lol) ${ }^{37}$

Ici, non seulement Univert liste un à un ses traits d'appartenance identitaire, en les organisant selon une certaine priorité, mais il ajoute un lol final qui semble souligner la bizarrerie de sa situation. Par ailleurs, il tient bien à marquer sa francité (« quebecofranco », « francais »), qu'il semble percevoir comme peu cohérente avec son activité de " gardener ».

Voyons maintenant quelles stratégies sont mises en œuvre dans le forum pour la construction d'une identité collective. L'emploi du nous se limite à un message d'organisation d'une action de la part d'Univert, où celui-ci construit son groupe encore virtuel (message suivi de la version anglaise qui a certainement été produite par un traducteur automatique) :

Argumentation et Analyse du Discours, 14 | 2015 
Donc, nous pouvons nous donner rendez-vous vers $19 \mathrm{~h} 30$ au centre du metro berriuqam (proche du banc rond noir). Je vais avoir un bulbe de tulipe dans mes mains. Nous vous attendons maximum 15 minutes de plus, avant de partir. Je ne sais pas encore exactement ou nous irons... Et si je susi [sic] seul... hey bien je serais seul... au mieux cela sera encore plus amusant.

Du point de vue énonciatif, ce message utilise alternativement un je, un nous et un vous, ce qui permet d'analyser la mise en discours des individus, de la collectivité et des relations entre ces deux instances. Le nous qui ouvre la partie soulignée de l'extrait témoigne du fait que l'auteur du message s'imagine déjà comme une collectivité («nous vous attendons ») dans laquelle le je du présent de l'énonciation va se fondre («Je ne sais pas encore où nous irons »). L'auteur du message ressent ensuite le besoin de protéger sa face en cas d'échec dans son projet de recrutement.

Si les exemples intéressants du point de vue de l'énonciation ne sont pas nombreux (le forum étant essentiellement le lieu virtuel où se fait la première prise de contact, et un type de texte dialogal, composé d'énoncés brefs à visée pragmatique, tournés vers le répertoire d'actions, voir Rennes 2011), il est possible d'y remarquer des locuteurs qui mettent en avant un ethos révolutionnaire, voire terroriste: «j'aimerais profiter de cette date du 8 octobre 2007 pour devenir officiellement un criminel vert », «It'd be nice to have a partner in crime ", « Vive la révolution! Vive la guérilla verte!", « Dans le cadre de la journée du 8 octobre comme "Day of The Heroic Guerrilla", je vous propose une ACTION de grâce. il serait intéressant de planter des bulbes de tulipes rouges dans des terrains publics». Cet ethos radical est ailleurs à la fois dit (contenu propositionnel : «[...] devenir officiellement un criminel vert») et montré (emploi du verbe ficher) : « je me fiche du cote esthétique de la chose. Je m'intéresse plutôt au cote potentiellement pratique (transformation de l'espace urbain "stérile" en espace de production de plantes comestibles) et social (rapprochement des citadins avec la production de leur nourriture, etc.)».

Alors que certains préparent leur « seed bombing " et du "stealth planting ", d'autres proposent de faire «quelques interventions sympathiques » dans leur «petite banlieue laide et monochrome ». Quelques-uns s'excusent aussi de mener des activités pas assez guerrières : "We're not being very "guerilla", and trying to obtain spaces with the permission of the administration. We are, however, doing a workshop on making seedbombs ». Or, par rapport aux textes médiatiques, le clivage scénographique ne semble pas ici recouper de façon aussi nette le clivage francophones/anglophones.

La question des motivations est également touchée: "Aussi qu'elles sont vos motivation de guerrieros jardinier: l'écologie, esthétique ou l'agroalimentaire urbain ?» - ce qui permet de vérifier que les activistes conceptualisent la GG comme un mouvement qui peut prendre des formes et avoir des orientations idéologiques différentes. Par ailleurs, un activiste se réclame de la mouvance Reynolds qui pratique la GG «propre » à l'anglaise («quand on fait du jardinage dans une ville c'est pour améliorer et non pour apporter un effet de laisser-aller, d'abandon, de négligé ») et déconseille à son interlocutrice d'utiliser les espèces envahissantes. Il apparait donc que jusque dans le choix des modalités pratiques d'intervention, le mouvement est diversifié. 


\subsection{Les pages Facebook et les blogues : nous et eux (stratégies de polarisation)}

Peut-être plus intéressantes se révèlent les pages web des groupes (Guerrilla Gardening Montréa ${ }^{38}$, Occupy Gardens Montréal ${ }^{39}$, Guerrilla Gardening dans le Mile End $^{40}$ ) et des activistes (blogue de Emily Rose Michaud: Le pouvoir aux pousses/Sprout out loud ${ }^{41}$ ): ceux-ci mettent en œuvre des stratégies rhétoriques de construction du groupe et d'incitation à la participation qui vont d'éléments formels tels que l'emploi de la rime ("jardiniers rebelles à vos truelles») et de l'allittération («free fresh food for all forever») ou du jeu sur les homophones («no peace without peas, peasful revolution »), à la construction de l'auditoire ("vous jardiniers clandestins », "vous jardiniers rebelles »), à l'affirmation de l'appartenance à un mouvement idéologique plus vaste -

Inspired by the Occupy Movement, Occupy Gardens represents the next step in our (vegetable) stand against inequality, corporate corruption and greed, growing hunger and the destruction of life itself for the sake of money (Occupy Gardens Montréal).

L'idéologie derrière l'idée: Favoriser l'agriculture et l'alimentation saine et locale, réapropriation de l'espace public par des initiatives citoyennes, réflexion sur le rythme de vie urbain, réutilisation de meubles trouvés à la poubelle, créer un climat convivial dans un espace urbain oublié [...] (Guerrilla Gardening dans le Mile End, souligné dans le texte) -

à l'inspiration à des valeurs partagées -

This is a resource/love based project

et au développement d'un sentiment communautaire d'inclusion :

We are in this together.

En revanche, la stigmatisation, voire l'identification d'un ennemi ne sont pas des pratiques courantes chez les activistes de la GG. La revendication s'exerce en effet contre des entités abstraites et générales :

Our industrial food system is rapidly depleting the Earth's arable soil and remaining forests, polluting our air, water and land. As Earth's population continues to grow we MUST invent alternative means of food production in order to feed the people and protect mother nature. A system motivated primarily by profit, without reference to human or environmental need, is doomed to failure. We must create a food system based upon love, cooperation and harmony with each other and the planet.

Pour conclure cette section consacrée à l'ethos auto-attribué, et reprendre les catégories proposées par Dominique Maingueneau et Ruth Amossy (1999), on constatera que finalement, l'ethos collectif discursif de la GG se manifeste plutôt du côté du dit que de celui du montré, sur la ligne continue qui les relie, même si, pour reprendre les propos de Maingueneau, « la distinction entre ethos dit et montré s'inscrit [...] aux extrêmes d'une ligne continue puisqu'il est impossible de définir une frontière nette entre le "dit" suggéré et le "montré" non "explicite" (Maingueneau 1999 : 91). En effet, dans mon corpus, la construction de l'ethos du mouvement de la GG se fait davantage par le biais d'outils déclaratifs et par le contenus propositionnel que par des stratégies énonciatives, à l'exception de la construction d'une scénographie qu'on pourrait appeler de « désobéissance civique ». Quant à l'ethos préalable ou prédiscursif, il s'agit d'un élément qui subit une évolution diachronique : en 2008 la GG était un mouvement presque inconnu, alors qu'aujourd'hui le citoyen sensible aux questions écologiques en a souvent une connaissance même très superficielle. De plus, certains 
éléments appartenant à la mémoire discursive sont au fur et à mesure intégrés à l'ethos collectif du mouvement dans un va-et-vient permanent entre prédiscursif et discursif. Une étude diachronique menée dans les années à venir sur l'évolution des discours de la GG et sur la GG, accompagnée d'une analyse ponctuelle des deux textes fondateurs de Richard Reynolds et David Tracey pourra surement nous éclairer davantage sur l'ethos du mouvement.

\section{Conclusion : la nébuleuse ethotique}

J'ai analysé dans cette contribution des textes numériques appartenant à des typologies textuelles différentes et provenant de sources énonciatives différentes, afin de proposer une réflexion sur l'ethos collectif de la GG. Nous avons observé tour à tour comment s'est construit dans les textes de ce corpus un ethos hétéro-attribué par le discours médiatique, qui s'articule avec un ethos prédiscursif ainsi qu'avec un ethos auto-attribué que l'on peut identifier dans les textes des activistes. Trois ordres de constatations s'imposent ici.

Premièrement, la polarisation entre la communauté francophone et la communauté anglophone est présente dans le discours médiatique francophone (ethos hétéroattribué), alors qu'il est absent des propos des activistes (ethos auto-attribué). Il est donc possible d'identifier à ce niveau-là une discordance entre les deux ethè qui demandera probablement des ajustements futurs si le mouvement s'implante de façon durable. Le corpus textuel produit par le mouvement est encore trop exigu pour qu'il soit possible de vérifier les rapports dialectiques qui pourront exister un jour entre l' ethos hétéro-attribué et l'ethos auto-attribué ainsi que le retravail du second par rapport au premier. De plus, les intérêts communicatifs des textes hétéro- et autoproduits divergent: alors que la presse francophone se penche sur les relations identitaires entre communauté francophone et GG en reléguant cette dernière en dehors ou aux marges de sa sphère collective, les activistes visent l'action et se montrent très collaboratifs d'une communauté à l'autre.

En second lieu, l'absence de véritable polarisation entre un nous et un eux (l'adversaire) confère au mouvement un ethos montré peu combattant, qui s'oppose à un ethos dit qui joue sur le clivage entre une scénographie de combattant, de guérillero, et une scénographie de pacifiste. En effet, dans les textes autoproduits, rares sont les exemples de violence verbale ou de comportement langagier agressif, même envers les adversaires. La scénographie guerrière se cantonne au champ sémantique de la guerre et aux citations de révolutionnaires et de stratèges célèbres. Si l'ethos dit reste en équilibre entre pacifisme et conflictualité, l'ethos montré reste résolument du côté du pacifisme et de l'inclusion : le groupe se veut solidaire à l'intérieur et ne veut pas faire peur à de potentielles nouvelles recrues. Le flirt avec la violence, l'illégalité, la clandestinité reste une manifestation apparemment superficielle.

51 En troisième lieu, et en conclusion, il nous paraît nécessaire de nous interroger sur la pertinence de la notion d'ethos pour ces mouvements antiautoritaires. Il appert à cette étape de ma recherche que, malgré le caractère "fluide » et mouvant de ce type de mouvement qui semble se soustraire à la constitution d'une identité forte de par son caractère anarchique, l'ethos en est malgré tout un élément de cohésion et de survie, partant nécessaire. 

si la notion d'identité se réfère à une mêmeté uniformisante, celle d'ethos collectif renvoie à une reconnaissance de soi dans le groupe, avec toutes ses nuances et sa complexité. Les termes du titre (conflictualité et inclusion) ne renvoient donc pas seulement à la question de la scénographie évoquée ci-dessus : ils renvoient aussi et surtout à l'image avec laquelle les militants du mouvement peuvent s'identifier, avec sa part de différences (qui peut générer une certaine conflictualitét2) et sa part d'inclusion (nécessaire à la cohésion, même faible, d'un mouvement). La « conceptual fuzzines » de la GG, «its relational and contextual nature [that] makes collapsing to a specific definition difficult " a déjà été soulignée par Annie Crane, Leela Viswanathan et Graham Whitelaw (2013: 76).

cen nébuleuse ethotique pour désigner l'ensemble des images de soi que l'orateur collectif projette dans son discours et qui sont à la base du pacte entre l'individu et l'instance collective dans laquelle il se reconnaît. La nébuleuse ethotique possède une forme incertaine, mouvante, en quelque sorte insaisissable, qui est le résultat de l'évolution gazeuse d'une étoile idéologique, lorsque celle-ci donne lieu non pas à une entité codifiée et structurée, mais à un ensemble de groupuscules se reconnaissant tous dans une idée centrale, la déclinant tour à tour différemment. La nébuleuse ethotique comprend l'ethos auto-attribué, bien sûr, mais aussi l'ethos hétéroattribué qui contribue, par réaction ou par assimilation, à l'enrichir et à la modifier.

Cette notion doit bien sûr être vérifiée, non seulement par une étude plus vaste sur la GG, mais aussi par des travaux portant sur d'autres mouvements sociaux ayant des caractéristiques semblables. Elle semble en effet pertinente pour les entités qui, tout en agissant dans l'ombre, de manière clandestine, doivent se construire une légitimation afin de gagner l'adhésion de nouveaux militants, se faire reconnaitre par les médias et se positionner par rapport à des mouvements qui s'en rapprochent idéologiquement et avec lesquels elles partagent des idéaux, des objectifs ou des procédés. La notion de nébuleuse ethotique pourra donc éviter une cristallisation excessive tout en permettant d'observer et de saisir la permanence de l'ethos collectif des mouvements souterrains dans des productions discursives hétérogènes, instables et discontinues.

\section{BIBLIOGRAPHY}

Amossy, Ruth (éd.). 1999. Images de soi dans le discours. La construction de l'ethos (Lausanne :

Delachaux \& Niestlé)

Amossy, Ruth. 1999. "L'ethos au carrefour des disciplines : rhétorique, pragmatique, sociologie des champs ", Ruth Amossy (éd.), Images de soi dans le discours. La construction de l'ethos (Lausanne : Delachaux \& Niestlé), 127-154

Amossy Ruth. 2010. La présentation de soi. Ethos et identité verbale (Paris : PUF)

Bulot, Thierry. 2001. «L'essence sociolinguistique des territoires urbains : un aménagement

linguistique de la ville? », Cahiers de Sociolinguistique 6, 5-11

Argumentation et Analyse du Discours, 14 | 2015 
Cassanas, Armelle, Aude Demange, Bénédicte Laurent \& Aude Lecler. 2003. Dialogisme et nomination (Montpellier : Publications Montpellier 3)

Charaudeau, Patrick. 2005. Le discours politique. Les masques du pouvoir (Paris : Vuibert)

Gardin, Bernard. 2002. « Locuteur collectif », Patrick Charaudeau \& Dominique Maingueneau (éds), Dictionnaire d'analyse du discours (Paris : Seuil), 352-353

Crane, Annie. 2011. Intervening with agriculture: a participatory action case study of guerrilla gardening in Kingston, Ontario (thèse de doctorat, Queen's University, Kingston, Canada)

Crane, Annie, Leela Viswanathan \& Graham Whitelaw. 2013. « Sustainability through Intervention : a Case Study of Guerrilla Gardening in Kingston, Ontario », Local Environment 18 : 1 , $71-90$

Delisle-L'Heureux, Nicolas \& Geneviève Lambert-Pilotte. 2010. « Une Révolution peut Commencer par un Seul Brin de Paille. Portrait de deux jardins autogérés de la grande région de Montréal ", Rapport de recherche par le Collectif de Recherche sur l'Autonomie Collective (CRAC) [en ligne : http://www.crac-kebec.org/files/Jardins_web5_0.pdf, consulté le 13 février 2014]

Elke, Laur. 2002. « Espaces linguistiques à Montréal », Marges Linguistiques 3, 137-150

Hou, Jeffrey. 2010. Insurgent public space: guerrilla urbanism and the remaking of contemporary cities (New York : Routledge)

Maingueneau, Dominique. 1999. «Ethos, scénographie, incorporation », Ruth Amossy (éd.), Images de soi dans le discours. La construction de l'ethos (Lausanne : Delachaux et Niestlé), 75-100

McClintock, Nathan. 2010. « Why Farm the City? Theorizing Urban Agriculture through a Lens of Metabolic Rift », Cambridge Journal of Region, Economics and Society $3: 2,191-207$

Orkibi, Eithan. 2008. «Ethos collectif et Rhétorique de polarisation : le discours des étudiants en France pendant la guerre d'Algérie ", Argumentation et Analyse du Discours 1 [en ligne : http:// aad.revues.org/438]

Orkibi, Eithan. 2012. Les étudiants de France et la guerre d'Algérie. Identité et expression collective de l'UNEF (1954-1962) (Paris : Syllepse)

Paveau, Marie-Anne. 2013. «Technodiscursivités natives sur Twitter. Une écologie du discours numérique », Fabien Liénard (éd.), Culture, identity and digital writing, Épistémè 9 (Séoul : Université de Korea), 139-176

Pinder, David. 2005. « Arts of Urban Exploration », Cultural Geographies 12 : 4, 383-411

Radice, Martha. 2000. Feeling Comfortable? The Urban Experience of Anglo-Montrealers (Sainte-Foy : Presses de l'Université Laval)

Raus, Rachele. 2007. « La terminologie des Bourses italienne et française : quelle relation à la langue anglaise ? », Irmtraud Behr, Dieter Hentschel, Michel Kauffmann \& Anja Kern (éds), Langue, économie et entreprise : le travail des mots (Paris : Presses de la Sorbonne Nouvelle), 311-325 Rennes, Juliette. 2011. « Les formes de la contestation. Sociologie des mobilisations et théories de l'argumentation ", À contrario 16, 151-173

Reynolds, Richard. 2008. On Guerilla Gardening (London : Bloomsbury Publishing), version française adaptée (2010) : La Guérilla jardinière (Paris : Yves Michel)

Ryan, Bartholomew A. 2010. Urban Agriculture from the Fringe: Pursuing a Greener Toronto (Kingston, Ontario : Master's Report for the School of Urban and Regional Planning, Queen's University) 
Sarrasin, Rachel, Anna Kruzynski, Sandra Jeppesen \& Émilie Breton. 2012. « Radicaliser l'action collective : portrait de l'option libertaire au Québec », Lien social et Politiques 68, 141-166

Simon, Sherry. 2006. Translating Montreal. Episodes in the Life of a Divided City (Montréal \& Kingston : McGill-Queen's University Press)

Tracey, David. 2007. Guerrilla Gardening : A Manualfesto, (Gabriola Island: New Society Publisher)

Zanetti, Olly. 2010. Guerrilla Gardening : Geographers and Gardeners, Actors and Networks :

Reconsidering Urban Public Space (Master's Dissertation, Queen Mary University of London)

\section{NOTES}

1. David Tracey se décrit sur son site (http://www.davidtracey.ca/) comme « a writer, designer and community ecologist based in Vancouver, Canada». Il est journaliste et fondateur de la société EcoUrbanist, spécialisée en aménagement environnemental. Guerrilla Gardening: A Manualfesto (2007) est son premier livre, suivi de Urban Agriculture: Ideas and Designs for the New Food Revolution (2011), The Earth Manifesto: Saving Nature with Engaged Ecology (2013) et deux ouvrages de fiction. Guerrilla Gardening : A Manualfesto est le premier manifeste du mouvement : il trace les origines et l'histoire de cette résistance écologique urbaine, en définit la philosophie et les objectifs, fournit des conseils pratiques pour les guérilleros et décrit de nombreux exemples d'actions et de projets déjà réalisés.

2. Richard Reynolds est un expert en horticulture britannique qui a fondé GuerrillaGardening.org en 2004. Son livre, très proche du texte de Tracey, insiste beaucoup moins sur l'aspect dangereux et illégal de la GG, tout en la définissant : « la culture illicite du terrain d'autrui » (Tracey 2010: 15). La deuxième différence importante consiste dans la dimension esthétique, très forte chez Reynolds : les actions de la GG doivent produire de la beauté et non pas du désordre.

3. Le travail de Crane est issu d'une recherche d'observation participante, car l'auteure fait partie du mouvement Dig Kingston. Ceci n'est pas sans poser la question délicate de la posture du chercheur.

4. Notamment Emily Rose Michaud et Peter Gibson (interviewé dans Tracey 2007).

5. Ce forum se trouve sur le site guerrillagardening.org, créé par Richard Reynolds. Il existe un forum pour chacune des villes où les activistes organisent des « attaques». Du moment que les forums sont classés par zones géographiques, il est significatif de constater que le forum montréalais est hébergé par le site « anglo-américain » (.org) et non pas par le site français (.fr).

6. https://www.facebook.com/occupygardensmontreal/info (page Facebook contenant le manifeste du groupe créée en 2011). Ce groupe est affilié à « Occupy gardens for world's peas ».

7. http://pousses.blogspot.it/ (consulté le 15 mars 2014).

8. Cet artiste n'a pas de site, mais l'on peut consulter à son sujet cet article paru dans Le Devoir du 6 mars 2014: http://www.ledevoir.com/culture/arts-visuels/153523/macadam-une-oasis-enplein-mile-end (consulté le 15 mars 2014).

9. Un acteur qui a participé à l'« Infringment festival »: http://www.infringementfestival.com/ lang/fr/ (consulté le 15 mars 2014).

10. http://roadsworth.com/home/ (consulté le 15 mars 2014).

11. http://ginabadger.ca/ (consulté le 15 mars 2014).

12. www.ecologieurbaine.net/promenade-Jane-2011-plateau-mont-royal (consulté le 15 mars 2014).

13. http://www.crapaud.uqam.ca/ et http://www.crac-kebec.org/ (consulté le 15 mars 2014).

14. http://www.cca-actions.org/fr/a-propos (consulté le 15 mars 2014). 